\title{
CONULL HYPERSURFACES IN MINKOWSKI SPACE
}

\author{
NORMAN GOLDSTEIN ${ }^{1}$
}

\begin{abstract}
A submanifold of $\mathbf{M}=\operatorname{Gr}\left(2, \mathbf{C}^{4}\right)$ is conull when its conormal space is in the kernel of the dualized conformal metric of $\mathbf{M}$. We show that there are no conull compact complex 3-dimensional submanifolds of $\mathbf{M}$.
\end{abstract}

Let $\mathbf{M}$ be complex Minkowski space, complex analytically homeomorphic to $\operatorname{Gr}\left(2, C^{4}\right)$, the Grassmannian of 2-planes in $\mathbf{C}^{4}$.

Each linear $\mathbf{P}^{2}$ in $\mathbf{M}$ is a null manifold i.e. the tangent space $T_{x}\left(\mathbf{P}^{2}\right)$ consists entirely of null vectors, for each $x \in \mathbf{P}^{2}$ cf. [4, 5.1]. ${ }^{2}$ Of course, any curve lying in such a $\mathbf{P}^{2}$ is null, but there are other null curves.

EXAMPLE 1.

$$
t \mapsto\left(\begin{array}{cccc}
1 & t & t^{2} & t^{4} \\
0 & 1 & 2 t & 4 t^{3}
\end{array}\right)
$$

is a null smooth $\mathbf{P}^{1}$ contained in $\mathbf{M}$, but is not contained in any hyperplane section via the Plucker embedding $\mathbf{M} \hookrightarrow \mathbf{P}^{5}$. (See Griffiths and Harris [2, 2.4] for a local description of null curves in Grassmannians.)

Let $X$ be a complex submanifold of $M$. We say that $X$ is conull if the conormal space of $X, N^{*} X \subset T^{*} \mathbf{M}$, consists entirely of null covectors. It is an exercise in linear algebra to verify that for $\operatorname{dim}(X)=2, X$ is null precisely when $X$ is conull. However, the plethora of null curves is in marked contrast to the lack of conull hypersurfaces.

THEOREM. Let $X$ be a smooth compact complex hypersurface of $\mathbf{M}$. Then $X$ is not conull.

Proof. Let $G=\operatorname{PGL}(3, \mathrm{C})$ be the projective linear group. The group $G$ acts transitively on $\mathbf{M}$, and defines an action on the cotangent bundle, $T^{*} \mathbf{M}$. This latter action has 3 orbits-the zero section, the null covectors, and the open orbit. It is well known that the normal bundle, $N X$, of $X$ in $\mathbf{M}$ is ample (e.g. [3, 2.9]). By [1, 4.5.2], $N^{*} X$ must meet the open orbit.

The theorem is not a local result.

Received by the editors May 7, 1981.

1980 Mathematics Subject Classification. Primary 32M10, 53C25; Secondary 32J25, 32M05.

Key words and phrases. Null and conull manifolds, Minkowski space, ample normal bundle, complex homogeneous space.

' Research partially supported by an NSERC postdoctoral fellowship. This paper was motivated by lectures given by R. O. Wells, Jr. at the NATO Advanced Study Institute of August 1980 at the Université de Montréal.

${ }^{2}$ The proof of the converse statement, as pointed out to me by Robert Bryant, is a straightforward local calculation: A null surface in $\mathbf{M}$ is necessarily a linear $\mathbf{P}^{2}$. 
EXAMPLE 2. Let $Y$ be the smooth points of a singular hyperplane section of $\mathbf{M}$. Then $Y$ is conull.

REMARK. The theorem is true with $M$ being any Grassmannian or quadric, and should remain valid with $M$ replaced by $G / P, G$ a simple complex Lie group and $P$ a maximal parabolic subgroup. A smooth hypersurface of $G / P$ does have an ample normal bundle, but I do not know, in general, whether $G / P$ is rigged, in the sense of $[1, \S 3]$.

\section{REFERENCES}

1. N. Goldstein, Ampleness in complex homogeneous spaces and a second Lefschetz theorem, preprint.

2. P. Griffiths and J. Harris, Algebraic geometry and local differential geometry, Ann. Sci. École Norm. Sup. 12 (1979), 355-432.

3. A. Papantonopoulou, Curves in Grassmann varieties, Nagoya Math. J. 66 (1977), 121-137.

4. R. O. Wells, Jr., Complex manifolds and mathematical physics, Bull. Amer. Math. Soc. (N.S.), 1 (1979), 296-336.

Mathematics Department, \# 121-1984 Mathematics RoAD, University of British Columbia, VANCOUVER, British Columbia V6T 1Y4, CaNADA

Current address: Department of Mathematics, Purdue University, West Layfayette, Indiana 47907 